\title{
On the boundedness of the Carleson operator near $L^{1}$
}

\author{
Victor Lie
}

\begin{abstract}
Based on the tile discretization elaborated in [14], we develop a Calderón-Zygmund type decomposition of the Carleson operator. As a consequence, through a unitary method that makes no use of extrapolation techniques, we recover previously known results regarding the largest rearrangement invariant space of functions with almost everywhere convergent Fourier series.
\end{abstract}

\section{Introduction}

In this paper we analyze some aspects of the behavior of the Carleson operator near $L^{1}$. Elaborating on an idea ${ }^{1}$ introduced in [14], we construct a CalderónZygmund type decomposition of the Carleson operator which, besides its own interest, may prove useful for studying other related problems. In particular, through this technique we are able to encompass previously known results regarding the problem of the largest rearrangement invariant space of (integrable) functions with almost everywhere convergent Fourier series. The relevant point here though is not the possibility of reproving these results but rather the existence of a method that avoids the limitations of extrapolation techniques - the main ingredient on which all the previous results rely.

As we will see, most of the difficulty and interest resides in the tile decomposition for the Carleson operator. Once this decomposition is available, everything else follows naturally. In the present paper we focus on the method rather than on obtaining the best possible space on which the Carleson operator is finitely almost everywhere. With respect to the latter objective, the best current result belongs to Arias de Reyna ([2]).

Mathematics Subject Classification (2010): Primary 42A20; Secondary 47A30.

Keywords: Time-frequency analysis, Carleson's theorem.

${ }^{1}$ This approach has as a consequence the removal of the exceptional sets in the tile decomposition and thus gives directly strong $L^{2}$ bounds for the Carleson operator. Also this is one of the key ingredients in providing the full range for the $L^{p}$ bounds $(1<p<\infty)$ of the polynomial Carleson operator. 
A significant improvement of his result seems to require an original idea, the present paper proposing just the first step towards opening up a new direction of investigation.

This being said, let us state the precise theme of interest regarding the behavior of Fourier series near $L^{1}$.

Open problem. What is the largest Banach rearrangement invariant space $\left(Y,\|\cdot\|_{Y}\right)$ with $Y \subseteq L^{1}(\mathbb{T})$ for which the Carleson operator defined ${ }^{2}$ by

$$
T: C^{\infty}(\mathbb{T}) \mapsto \mathcal{D}^{\prime}(\mathbb{T})
$$

with

$$
T f(x):=\sup _{N \in \mathbb{N}}\left|\int_{\mathbb{T}} e^{i N(x-y)} \cot (x-y) f(y) d y\right|,
$$

obeys the relation

$$
\|T f\|_{1, \infty} \lesssim\|f\|_{Y} \quad \forall f \in Y ?
$$

Observation. Notice that by Stein's maximal principle ([19]) the above open problem is equivalent to asking for the largest Banach rearrangement invariant space $\left(Y,\|\cdot\|_{Y}\right)$ with $Y \subseteq L^{1}(\mathbb{T})$ for which the partial Fourier series $\left\{S_{n} f\right\}_{n}$ have the property

$$
S_{n} f(x) \stackrel{n \rightarrow \infty}{\longrightarrow} f(x) \quad \text { a.e. } x \in \mathbb{T}, \forall f \in Y .
$$

A lot has been written on this subject. The major breakthrough was made by Carleson $([6])$, who showed that $L^{2}(\mathbb{T}) \subset Y$. Later, Hunt ([10]) extended this result by showing that $L^{p}(\mathbb{T}) \subset Y$ for any $1<p<\infty$. A new influential proof of Carleson's result ${ }^{3}$ was given by Fefferman in [9]. From this point, the problem evolved at a slower rate towards the limiting index $p=1$. Sjölin proved in [16] that one may take $Y=L(\log L)^{2}$ in (1.2) (if one requires strong $L^{1}$ bounds in (1.2)) or even $L \log L \log \log L$ for just $L^{1, \infty}$ bounds. Next, Soria ([17], [18]) constructed a larger space $B_{\varphi_{1}}^{*} \subset Y$. In [1], Antonov showed that (1.2) holds for $Y=L \log L \log \log \log L$. Finally, combining elements from Antonov's and Soria's approaches with techniques on logconvex quasi-Banach spaces, Arias de Reyna ([2]) proved that $Q A \subset Y$, where $Q A$ is a quasi-Banach space described in the appendix.

The following chain of inclusions ${ }^{5}$ holds:

(1.4) $L(\log L)^{2} \subsetneq L \log L \log \log L \subsetneq B_{\varphi_{1}}^{*}, L \log L \log \log \log L \subsetneq Q A \subsetneq L \log L$

The main result in this paper is given by:

\footnotetext{
${ }^{2}$ We write $D^{\prime}(\mathbb{T})$ for the class of distributions supported on the torus.

${ }^{3}$ More recently ([12]), Lacey and Thiele, combining ideas from both [6] and [9], provided a third approach to Carleson's theorem on the pointwise convergence of the Fourier series.

${ }^{4}$ See the appendix for the definition.

${ }^{5}$ Notice that there is no order relation between the spaces $B_{\varphi_{1}}^{*}$ and $L \log L \log \log \log L$ (for more details see [2]).
} 
Theorem 1.1. There exists a partition of the family of tiles

$$
\mathbb{P}=\bigcup_{n \in \mathbb{N}} \mathbb{P}_{n}, \quad \text { with } \quad T=\sum_{n} T^{\mathbb{P}_{n}}
$$

such that for each $n \in \mathbb{N}$ we have:

a) Given nonzero $f \in L^{1}$, there exists a further decomposition ${ }^{6}$

$$
\mathbb{P}_{n}=\bigcup_{\alpha \in \mathbb{Z}} \mathbb{P}_{n}^{\alpha}
$$

such that for any $n \in \mathbb{N}$ we have ${ }^{7}$

$$
\begin{aligned}
\operatorname{supp} T^{\mathbb{P}_{n}^{\alpha}} \subseteq 100\left\{M f>2^{-\alpha}\right\}, \\
\left\|T^{\mathbb{P}_{n}^{\alpha}} f\right\|_{1} \lesssim 2^{-\alpha}\left|\left\{M f>2^{-\alpha}\right\}\right|,
\end{aligned}
$$

where here $M$ stands for the dyadic Hardy-Littlewood maximal function.

In particular, we deduce that

$$
\left\|T^{\mathbb{P}_{n}} f\right\|_{1} \lesssim\|f\|_{L \log L}
$$

b) For $f \in L^{1}$ there holds:

$$
\left\|T^{\mathbb{P}_{n}} f\right\|_{1, \infty} \lesssim\|f\|_{1}
$$

c) If $1<p<\infty$ and $p^{*}=\min \left\{p, p^{\prime}\right\}$, with $p^{\prime}$ the Hölder conjugate of $p$, then there exists an absolute constant $\delta>0$ such that

$$
\left\|T^{\mathbb{P}_{n}} f\right\|_{p} \lesssim p 2^{-\delta n\left(1-1 / p^{*}\right)}\|f\|_{p} .
$$

d) If $f \in L^{p}$ with $1<p \leq \infty$ then $^{8}$

$$
\|T f\|_{1, \infty} \lesssim p\|f\|_{1} \log \frac{2\|f\|_{p}}{\|f\|_{1}} .
$$

As a direct application of Theorem 1.1 we have the following:

Corollary 1.2. The following are true:

1) (Carleson-Hunt, [6], [10]) $\|T f\|_{p} \lesssim p\|f\|_{p}$ for any $1<p<\infty$.

2) (Sjölin, [16]) $\|T f\|_{1} \lesssim\|f\|_{L(\log L)^{2}}$.

3) For measurable $E \subseteq[0,1],\left\|T \chi_{E}\right\|_{1, \infty} \lesssim|E| \log (2 /|E|)$.

\footnotetext{
${ }^{6}$ This second decomposition depends on $f$.

${ }^{7}$ Here, if $J=(c-|J| / 2, c+|J| /|2|)$ is any given interval, we use the standard notation $b J$ $(b>0)$ to designate the interval $(c-b|J| / 2, c+b|J| /|2|)$. Moreover, if $I=\cup_{n \in \mathbb{N}} J_{n}$ with each $J_{n}$ an interval, then we set $b I:=\cup_{n \in \mathbb{N}} b J_{n}$.

${ }^{8}$ Throughout the paper, for $x>0$, we set $\log x:=\log _{2} x$.
} 
4) (Sjölin, [16]) $\|T f\|_{1, \infty} \lesssim\|f\|_{L \log L \log \log L}$.

5) (Arias de Reyna, [2]) $\|T f\|_{1, \infty} \lesssim\|f\|_{Q A}$. In particular 5) also implies

6) (F. Soria, [17],[18]) $\|T f\|_{1, \infty} \lesssim\|f\|_{B_{\varphi_{1}}^{*}}$.

7) (Antonov, [1]) $\|T f\|_{1, \infty} \lesssim\|f\|_{L \log L \log \log \log L}$.

Comment. In fact, as a consequence of d) in Theorem 1.1, we obtain that for $1<p \leq \infty$ one has

$$
\|T f\|_{1, \infty} \lesssim p\|f\|_{Q A_{p}},
$$

where $Q A_{p}$ is the quasi-Banach space defined by

$$
Q A_{p}:=\left\{f: \mathbb{T} \mapsto C \mid f \text { measurable, }\|f\|_{Q A_{p}}<\infty\right\}
$$

with

$$
\|f\|_{Q A_{p}}:=\inf \left\{\sum_{j=1}^{\infty}(1+\log j)\left\|f_{j}\right\|_{1} \log \frac{2\left\|f_{j}\right\|_{p}}{\left\|f_{j}\right\|_{1}}\left|f=\sum_{j=1}^{\infty} f_{j}, \sum_{j=1}^{\infty}\right| f_{j} \mid<\infty \text { a.e. }\right\} .
$$

However, it turns out, based on an observation of Luis Rodríguez-Piazza kindly communicated to me by Arias de Reyna, that the spaces $Q A_{p}$ are equivalent in the sense that

$$
\|f\|_{Q A_{p}} \approx_{p}\|f\|_{Q A_{\infty}}
$$

Notice that $Q A_{\infty}$ is the original space $Q A$ defined in [2]; for an interesting study concerning the properties of the space $Q A$ one should consult [7].

Acknowledgment. I thank Arias de Reyna for reading the manuscript and supplying useful comments.

\section{Discretization of the operator}

In this section we decompose the operator $T$ in components $\left\{T_{P}\right\}_{P}$ which are "well" time-frequency localized. We follow the procedure in [9].

Let $T$ be the Carleson operator defined by

$$
T f(x):=\sup _{N \in \mathbb{R}}\left|\int_{\mathbb{T}} \frac{1}{x-y} e^{i N(x-y)} f(y) d y\right|,
$$

which after linearization becomes

$$
T f(x)=\int_{\mathbb{T}} \frac{1}{x-y} e^{i N(x)(x-y)} f(y) d y,
$$

where $N$ is some arbitrary measurable function (which henceforth will be fixed).

Choose now an odd $C^{\infty}$ function $\psi$ such that

$$
\operatorname{supp} \psi \subseteq\{y \in \mathbb{R}|2<| y \mid<8\}
$$


and having the property

$$
\frac{1}{y}=\sum_{k \geq 0} \psi_{k}(y) \quad \forall 0<|y|<1,
$$

where by definition $\psi_{k}(y):=2^{k} \psi\left(2^{k} y\right)($ with $k \in \mathbb{N})$.

Thus, we have that

$$
T f(x)=\sum_{k \geq 0} T_{k} f(x):=\sum_{k \geq 0} \int_{\mathbb{T}} e^{i N(x) y} \psi_{k}(y) f(x-y) d y .
$$

Take the canonical dyadic grid on $\mathbb{T}$ (time grid), denoted by $\mathcal{D}_{\mathcal{T}}$, and the corresponding canonical dyadic grid on $\mathbb{R}$ (frequency grid), denoted by $\mathcal{D}_{\mathcal{F}}$. A tile $P$ will consist of a $\operatorname{pair}^{9}[\omega, I] \in \mathcal{D}_{\mathcal{F}} \times \mathcal{D}_{\mathcal{T}}$ with the property that $|\omega|=|I|^{-1}$. The collection of all such tiles will be denoted by $\mathbb{P}$. Further, for each $P=[\omega, I] \in \mathbb{P}$ we set $E(P):=\{x \in I \mid N(x) \in \omega\}$.

For $|I|=2^{-k}(k \geq 0)$ and $P=[\omega, I] \in \mathbb{P}$, we define the operator $T_{P}$ on $L^{2}(\mathbb{T})$ by

$$
T_{P} f(x)=\left\{\int_{\mathbb{T}} e^{i N(x) y} \psi_{k}(y) f(x-y) d y\right\} \chi_{E(P)}(x) .
$$

Notice that the Carleson operator satisfies

$$
T f(x)=\sum_{P \in \mathbb{P}} T_{P} f(x) .
$$

Finally, whenever $\mathcal{P} \subseteq \mathbb{P}$ is a family of tiles we set

$$
T^{\mathcal{P}}:=\sum_{P \in \mathcal{P}} T_{P}
$$

\section{The proof of Corollary 1.2}

\subsection{The proof of 1 )}

We want to show that, for $1<p<\infty$,

$$
\|T f\|_{p} \lesssim p\|f\|_{p} .
$$

This is a trivial application of statement c) in Theorem 1.1. Indeed, we have

$$
\|T f\|_{p} \leq \sum_{n \in N}\left\|T^{\mathbb{P}_{n}} f\right\|_{p} \lesssim p \sum_{n \in \mathbb{N}} 2^{-\delta n\left(1-1 / p^{*}\right)}\|f\|_{p} \lesssim\|f\|_{p} .
$$

\footnotetext{
${ }^{9}$ Here we abuse language and refer to $\mathcal{D}_{\mathcal{T}}$ also as the collection of all dyadic intervals for the specified grid. We do the same for $\mathcal{D}_{\mathcal{F}}$.
} 


\subsection{The proof of 2 )}

We want to show that

$$
\|T f\|_{1} \lesssim\|f\|_{L(\log L)^{2}}
$$

We will use the decomposition defined for each $l \in \mathbb{Z}$ by

$$
Q_{l}:=\left\{x \in \mathbb{T}|| f(x) \mid \in\left[2^{l}, 2^{l+1}\right)\right\} .
$$

Then we have that

$$
\|f\|_{L(\log L)^{2}} \sim \sum_{l \in \mathbb{Z}} 2^{l}\left|Q_{l}\right|\left(\log \frac{2}{\left|Q_{l}\right|}\right)^{2} .
$$

Thus, using duality, to prove (3.2) it will be enough to show

$$
\int_{Q_{l}}\left|T^{*} g\right| \lesssim\left|Q_{l}\right|\left(\log \frac{2}{\left|Q_{l}\right|}\right)^{2}\|g\|_{\infty} .
$$

Taking $f=\chi_{Q_{l}}=\chi_{Q}$ in the statements dual to a) and c) in Theorem 1.1 we deduce

$$
\int_{Q}\left|T^{\mathbb{P}_{n} *} g\right| \lesssim|Q| \log \frac{2}{|Q|}\|g\|_{\infty} \quad \text { and } \quad \int_{Q}\left|T^{\mathbb{P}_{n}^{*}} g\right| \lesssim|Q|^{1 / 2} 2^{-n \delta / 2}\|g\|_{\infty} .
$$

Thus

$$
\int_{Q}\left|T^{\mathbb{P}_{n}{ }^{*}} g\right| \lesssim \sum_{n} \min \left\{|Q| \log \frac{2}{|Q|},|Q|^{1 / 2} 2^{-n \delta / 2}\right\}\|g\|_{\infty} \lesssim|Q|\left(\log \frac{2}{|Q|}\right)^{2}\|g\|_{\infty} .
$$

\subsection{The proof of 3 )}

To show

$$
\left\|T \chi_{E}\right\|_{1, \infty} \lesssim|E| \log \frac{2}{|E|}
$$

just apply d) with $f=\chi_{E}$.

\subsection{The proof of 4)}

Of course, this result is implied by claims 5)-7). Still, we think it is worth providing a different approach to this problem, one that isolates a relevant idea in Sjölin's original proof and adapts it to the context of Theorem 1.1.

Our task is to prove that

$$
\|T f\|_{1, \infty} \lesssim\|f\|_{L \log L \log \log L}
$$

where here $\|\cdot\|_{L \log L \log \log L}$ is defined according to (8.2) in the Appendix.

The definition that we take here for $L \log L \log \log L$ is as in the original paper of Sjölin ([16]) given by the space of functions $f \in L^{1}(\mathbb{T})$ for which we have ${ }^{10}$ $\|f\|_{\mathcal{L} \log \mathcal{L} \log \log \mathcal{L}}=\int|f| \log _{+}|f| \log _{+} \log _{+}|f|<\infty$.

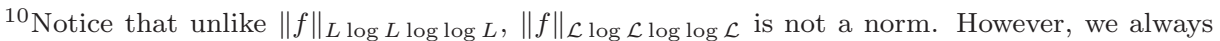

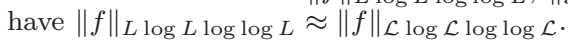


Thus, setting as before $Q_{l}:=\left\{x \in \mathbb{T}:|f(x)| \approx 2^{l}\right\}$, we can always assume $Q_{l}=\emptyset$ for $l \leq 2$ and consequently

$$
\|f\|_{\mathcal{L} \log \mathcal{L} \log \log \mathcal{L}} \approx \sum_{l>2} 2^{l} l \log l\left|Q_{l}\right|
$$

Now fix $l>2$. For $\chi_{l}=\chi_{Q_{l}}$ we apply ${ }^{11}$ the tile partition described in a) of Theorem 1.1, taking into account that $\left\|\chi_{l}\right\|_{\infty} \leq 1$. Then for each $n \in \mathbb{N}$ there exists a decomposition of

$$
\mathbb{P}_{n}=\bigcup_{\alpha \in \mathbb{N}} \mathbb{P}_{n}^{\alpha}
$$

such that

(3.7) $\operatorname{supp} T^{\mathbb{P}_{n}^{\alpha}} \subseteq 100\left\{M \chi_{l}>2^{-\alpha}\right\} \quad$ and $\quad\left\|T^{\mathbb{P}_{n}^{\alpha}} \chi_{l}\right\|_{1} \lesssim 2^{-\alpha}\left|\left\{M \chi_{l}>2^{-\alpha}\right\}\right|$.

Partition the set $\mathbb{N}=A_{l} \cup B_{l} \cup C_{l}$, where

$$
\begin{aligned}
& A_{l}:=\left\{r \in \mathbb{N}: 2^{-r}<\gamma 2^{-l} l^{-3}\right\}, \\
& B_{l}:=\left\{r \in \mathbb{N}: \gamma 2^{-l} l^{-3} \leq 2^{-r}<\gamma 2^{-l}\right\}, \\
& C_{l}:=\left\{r \in \mathbb{N}: 2^{-r} \geq \gamma 2^{-l}\right\},
\end{aligned}
$$

with $\gamma>0$ a parameter that will be chosen later. Set

$$
T^{\mathbb{P}^{\alpha}}:=\sum_{n \in \mathbb{N}} T^{\mathbb{P}_{n}^{\alpha}}
$$

Then, by (3.7), we have

$$
\operatorname{supp} T^{\mathbb{P}^{\alpha}} \subseteq 100\left\{M \chi_{l} \geq 2^{-\alpha}\right\}
$$

which implies that

$$
\sum_{\alpha \in C_{l}}\left|\operatorname{supp} T^{\mathbb{P}^{\alpha}}\right| \lesssim \sum_{\alpha \in C_{l}}\left|\left\{M \chi_{l} \geq 2^{-\alpha}\right\}\right| \lesssim \sum_{\alpha \in C_{l}} 2^{\alpha}\left|Q_{l}\right| \lesssim \gamma^{-1} 2^{l}\left|Q_{l}\right|
$$

Thus the set $S_{l}=\bigcup_{\alpha \in C_{l}} \operatorname{supp} T^{\mathbb{P}^{\alpha}}$ can be excised since we have good control on

$$
\sum_{l \in \mathbb{Z}}\left|S_{l}\right| \lesssim \gamma^{-1} \sum_{l \in \mathbb{Z}} 2^{l}\left|Q_{l}\right| \lesssim \gamma^{-1}\|f\|_{\mathcal{L} \log \mathcal{L} \log \log \mathcal{L} .}
$$

Next, it is useful to notice that by (3.7) we have

$$
\left\|T^{\mathbb{P}_{n}^{\alpha}} \chi_{l}\right\|_{1} \lesssim 2^{-\alpha}\left|\left\{M \chi_{l} \geq 2^{-\alpha}\right\}\right| \lesssim \min \left\{2^{-\alpha},\left|Q_{l}\right|\right\},
$$

while from d) in Theorem 1.1 we infer that

$$
\left\|T^{\mathbb{P}_{n}^{\alpha}} \chi_{l}\right\|_{2} \lesssim 2^{-n \delta / 2}\left|Q_{l}\right|^{1 / 2} .
$$

From these we deduce

$$
\left\|T^{\mathbb{P}^{\alpha}} \chi_{l}\right\|_{1} \lesssim \alpha \min \left\{2^{-\alpha},\left|Q_{l}\right|\right\}
$$

\footnotetext{
${ }^{11}$ We assume here that $\left|Q_{l}\right| \neq 0$.
} 
Then, from (3.10), we have that

$$
\sum_{\alpha \in B_{l}}\left\|T^{\mathbb{P}^{\alpha}} \chi_{l}\right\|_{1} \lesssim\left(l+\log \frac{1}{\gamma}\right) \log l\left|Q_{l}\right|,
$$

and similarly,

$$
\sum_{\alpha \in A_{l}}\left\|T^{\mathbb{P}^{\alpha}} \chi_{l}\right\|_{1} \lesssim \sum_{\alpha \in A_{l}} \alpha 2^{-\alpha} \lesssim \gamma\left(1+\log \frac{1}{\gamma}\right) 2^{-l} l^{-2}
$$

Combining (3.9), (3.11) and (3.12) and choosing $\gamma=c\|f\|_{\mathcal{L} \log \mathcal{L} \log \log \mathcal{L}}^{2 / 3}$ (with $c>0$ some large number) we have proved the following:

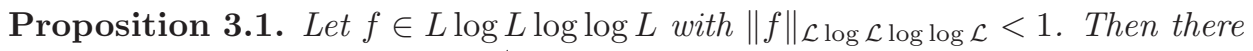
exists $A \subseteq[0,1]$ with $|A| \leq\|f\|_{\mathcal{L} \log \mathcal{L} \log \log \mathcal{L}}^{1 / 3}$ and an absolute constant $C>0$ such that

$$
\|T f\|_{L^{1}\left(A^{c}\right)} \leq C\|f\|_{\mathcal{L} \log \mathcal{L} \log \log \mathcal{L}}^{1 / 2}
$$

Now, by a canonical density argument, we obtain that the sequence of the partial Fourier sums $\left\{S_{n} f(x)\right\}_{n}$ converges almost everywhere for $f \in L \log L \log \log L$. The relation (3.6) follows from an application of Stein's maximal principle ([19]).

\subsection{The proof of 5)-8)}

We will show that

$$
\|T f\|_{1, \infty} \lesssim p\|f\|_{Q A_{p}}
$$

We choose ${ }^{12}$ here to use the log-convexity result due to Kalton ([11]), as further described in [2]:

Theorem (Kalton, [11]). If $\left\{f_{j}\right\}_{j \geq 1}$ is a sequence of functions in $L^{1, \infty}(\mathbb{T})$, then

$$
\left\|\sum_{j=1}^{\infty} f_{j}\right\|_{1, \infty} \lesssim \sum_{j=1}^{\infty}(1+\log j)\left\|f_{j}\right\|_{1 \infty}
$$

Take $f \in \mathcal{W}$ and set $f=\sum_{j=1}^{\infty} f_{j}$ with $f_{j}$ as in (1.11). Apply now (3.15) and point d) in Theorem 1.1 to conclude

$$
\|T f\|_{1, \infty} \lesssim \sum_{j}(1+\log j)\left\|T f_{j}\right\|_{1 \infty} \lesssim p \sum_{j}(1+\log j)\left\|f_{j}\right\|_{1} \log \frac{2\left\|f_{j}\right\|_{p}}{\left\|f_{j}\right\|_{1}} .
$$

\footnotetext{
${ }^{12}$ One can use a different approach to show (3.14) that avoids the use of Kalton's theorem on the log convexity of $\|\cdot\|_{1, \infty}$. For this, one can follow the proof of 4 ).
} 


\section{Finding structures in our family of tiles; main definitions}

In this section we isolate the main concepts needed for further discretizing and organizing the family $\mathbb{P}$ of tiles. Our presentation here is based on the definitions and notations introduced in [14].

Definition 4.1 (weighting the tiles). Let $\mathcal{A}$ be a (finite) union of dyadic intervals in $[0,1]$ and let $\mathcal{P}$ be a finite family of tiles. For $P=[\omega, I] \in \mathcal{P}$ with $I \subseteq \mathcal{A}$ we define the mass of $P$ relative to the set of tiles $\mathcal{P}$ and the set $\mathcal{A}$ as

$$
A_{\mathcal{P}, \mathcal{A}}(P):=\sup _{\substack{P^{\prime}=\left[\omega^{\prime}, I^{\prime}\right] \in \mathcal{P} \\ I \subseteq I^{\prime} \subseteq \mathcal{A}}} \frac{\left|E\left(P^{\prime}\right)\right|}{\left|I^{\prime}\right|}\left\lceil\Delta\left(10 P, 10 P^{\prime}\right)\right\rceil^{N},
$$

where $N$ is a fixed large natural number.

Definition 4.2 (ordering the tiles). Let $P_{j}=\left[\omega_{j}, I_{j}\right] \in \mathbb{P}$ with $j \in\{1,2\}$. We say that $P_{1} \leq P_{2}$ if $I_{1} \subseteq I_{2}$ and $\omega_{1} \supseteq \omega_{2}$. We write $P_{1}<P_{2}$ if $P_{1} \leq P_{2}$ and $\left|I_{1}\right|<\left|I_{2}\right|$.

Notice that $\leq$ defines an order relation on the set $\mathbb{P}$.

Definition 4.3 (modulated/scaled (maximal) Hilbert transform - "tree"). We say that a set of tiles $\mathcal{P} \subset \mathbb{P}$ is a tree with top $P_{0}$ if the following conditions hold:

1) For all $P \in \mathcal{P}$ there holds $2 P \leq 10 P_{0}$.

2) If $P=\left[\omega_{1}, I_{P}\right] \in \mathcal{P}$ and $P^{\prime}=\left[\omega_{2}, I_{P}\right]$ are such that $2 P^{\prime} \leq P_{0}$, then $P^{\prime} \in \mathcal{P}$.

3) If $P_{1}, P_{2} \in \mathcal{P}$ and $P_{1} \leq P \leq P_{2}$, then $P \in \mathcal{P}$.

Definition 4.4 (Carleson measure relative to a tree). We say that a set of tiles $\mathcal{P} \subset \mathbb{P}$ is a sparse tree if $\mathcal{P}$ is a tree and for any $P \in \mathcal{P}$ we have

$$
\sum_{\substack{P^{\prime} \in \mathcal{P} \\ I_{P^{\prime}} \subseteq I_{P}}}\left|I_{P^{\prime}}\right| \leq C\left|I_{P}\right|
$$

where here $C>0$ is an absolute constant.

Definition 4.5 ( $L^{\infty}$ control over union of trees). Fix $n \in \mathbb{N}$. We say that $\mathcal{P} \subseteq \mathbb{P}_{n}$ is an $L^{\infty}$-forest (of the $n^{\text {th }}$-generation) if

i) $\mathcal{P}$ is a collection of separated trees, i.e.,

$$
\mathcal{P}=\bigcup_{j \in \mathbb{N}} \mathcal{P}_{j}
$$

with each $\mathcal{P}_{j}$ a tree with top $P_{j}=\left[\omega_{j}, I_{j}\right]$ and such that

$$
\forall k \neq j \& \forall P \in \mathcal{P}_{j} \quad 2 P \not 10 P_{k} ;
$$


ii) the counting function

$$
\mathcal{N}_{\mathcal{P}}(x):=\sum_{j} \chi_{I_{j}}(x)
$$

obeys the estimate $\left\|\mathcal{N}_{\mathcal{P}}\right\|_{L^{\infty}} \lesssim 2^{n}$.

Further, if $\mathcal{P} \subseteq \mathbb{P}_{n}$ only consists of sparse separated trees then we refer to $\mathcal{P}$ as a sparse $L^{\infty}$-forest.

Definition 4.6 (BMO control over union of trees). A set $\mathcal{P} \subseteq \mathbb{P}_{n}$ is called a BMOforest (of the $n^{\text {th }}$-generation), or simply a forest, if

i) $\mathcal{P}$ may be written as

$$
\mathcal{P}=\bigcup_{j \in \mathbb{N}} \mathcal{P}_{j}
$$

with each $\mathcal{P}_{j}$ an $L^{\infty}$-forest (of the $n^{\text {th }}$-generation);

ii) for any $P \in \mathcal{P}_{j}$ and $P^{\prime} \in \mathcal{P}_{k}$ with $j, k \in \mathbb{N}, j<k$ we either have $I_{P} \cap I_{P^{\prime}}=\emptyset$ or

$$
\left|I_{P^{\prime}}\right| \leq 2^{j-k}\left|I_{P}\right|
$$

As before, if $\mathcal{P} \subseteq \mathbb{P}_{n}$ only consists of sparse $L^{\infty}$-forest, then we refer to it as a sparse forest.

Notice that if $\mathcal{P} \subseteq \mathbb{P}_{n}$ is a forest then, by ii) above, the counting function $\mathcal{N}_{\mathcal{P}}:=\sum_{j} \mathcal{N}_{\mathcal{P}_{j}}$ obeys the estimate

$$
\left\|\mathcal{N}_{\mathcal{P}}\right\|_{\mathrm{BMO}_{C}} \lesssim 2^{n}
$$

\section{Discretization of the family of tiles}

\subsection{The mass decomposition: $n$ discretization}

In this section we partition the set $\mathbb{P}$ into $\bigcup_{n \in \mathbb{N}} \mathbb{P}_{n}$, with each $\mathbb{P}_{n}$ a BMO-forest. The procedure described below is an adaptation of the one introduced by the author in [14] for proving the $L^{p}$ boundedness $(1<p<\infty)$ of the polynomial Carleson operator.

We start by constructing the family $\mathbb{P}_{1}$ according to the following algorithm:

- Let $\mathcal{P}_{1}^{0, m a x}$ be the collection of maximal tiles $P \in \mathbb{P}$ with $|E(P)| /|I| \geq 1 / 2$ and let $\mathcal{I}_{1}^{0}:=\left\{I \mid P=[\omega, I] \in \mathcal{P}_{1}^{0, \max }\right\}$.

- Define the counting function $\mathcal{N}_{1}^{0}:=\sum_{I \in \mathcal{I}_{1}^{0}} \chi_{I}$ and verify that

$$
\left\|\mathcal{N}_{1}^{0}\right\|_{\mathrm{BMO}_{C}}:=\sup _{\substack{J \text { dyadic } \\ J \subseteq[0,1]}} \frac{\sum_{\substack{I \subseteq J \\ I \in \mathcal{I}_{1}^{0}}}|I|}{|J|} \leq 2 .
$$

and hence $\mathcal{N}_{1}^{0} \in \mathrm{BMO}_{D}(\mathbb{R})$. 
- Apply the John-Nirenberg inequality

$$
\left|\left\{x \in J:\left|\mathcal{N}_{1}(x)-\frac{1}{|J|} \int_{J} \mathcal{N}_{1}^{0}\right|>\gamma\right\}\right| \lesssim|J| \exp \left(-c \frac{\gamma}{\left\|\mathcal{N}_{1}^{0}\right\|_{\mathrm{BMO}_{D}(\mathbb{R})}}\right) .
$$

for $^{13} \gamma>c\left\|\mathcal{N}_{1}^{0}\right\|_{\mathrm{BMO}_{C}}$ (here $c>0$ is an appropriately chosen large absolute constant) and deduce that

$$
\left|\left\{x \in J: \sum_{\substack{I \subseteq J \\ I \in \mathcal{I}_{1}^{0}}} \chi_{I}(x)>\gamma\right\}\right| \lesssim|J| e^{-c} .
$$

- Based on (5.3), conclude that the set

$$
A_{1}^{1}:=\left\{x \in[0,1]: \sum_{\substack{I \subseteq[0,1] \\ I \in \mathcal{I}_{1}^{0}}} \chi_{I}(x)>c\left\|\mathcal{N}_{1}^{0}\right\|_{\mathrm{BMO}_{C}}\right\}
$$

obeys the relation $\left|A_{1}^{1}\right| \leq e^{-c}$.

- Remove from $\mathbb{P}$ all the tiles for which the time interval is not included in the set $A_{1}^{1}$. Run the above algorithm again for the new collection $\mathbb{P}$. This process ends in a finite number of steps since without loss of generality we may assume that the initial family $\mathbb{P}$ is finite.

This way, after the $k^{\text {th }}$ repetition of our algorithm, we have constructed the sets $A_{1}^{k}, \mathcal{P}_{1}^{k, \max }, \mathcal{I}_{1}^{k}$ and the counting function $\mathcal{N}_{1}^{k}$.

We now define the 1-maximal set of tiles $\mathcal{P}_{1}^{\max }:=\bigcup_{k} \mathcal{P}_{1}^{k, \max }$, the collection of the time intervals $\mathcal{I}_{1}:=\bigcup_{k} \mathcal{I}_{1}^{k}$ and finally the counting function $\mathcal{N}_{1}:=\sum_{I \in \mathcal{I}_{1}} \chi_{I}$.

Notice that from the above construction we have that

- $\left\|\mathcal{N}_{1}\right\|_{\mathrm{BMO}_{C}} \lesssim \max _{k}\left\|\mathcal{N}_{1}^{k}\right\|_{\mathrm{BMO}_{C}}$;

- for any $l<k$ we have $A_{1}^{k} \subset A_{1}^{l}$ and $\left|A_{1}^{k}\right| \leq e^{-(k-l) c}\left|A_{1}^{l}\right|$.

Next, define

$$
\mathcal{P}_{1}^{0}:=\left\{P=[\omega, I] \in \mathbb{P}: \begin{array}{c}
A_{\mathbb{P},[0,1]}(P) \in\left[2^{-1}, 2^{0}\right], \\
\text { and if } I \subseteq A_{1}^{1} \text { then } A_{\mathbb{P}, A_{1}^{1}}(P) \notin\left[2^{-1}, 2^{0}\right]
\end{array}\right\}
$$

and further, by induction, construct

$$
\mathcal{P}_{1}^{k}:=\left\{P=[\omega, I] \in \mathbb{P}: \begin{array}{c}
I \subseteq A_{1}^{k}, A_{\mathbb{P}, A_{1}^{k}}(P) \in\left[2^{-1}, 2^{0}\right) \\
\text { and if } I \subseteq A_{1}^{k+1} \text { then } A_{\mathbb{P}, A_{1}^{k+1}}(P) \notin\left[2^{-1}, 2^{0}\right]
\end{array}\right\} .
$$

Finally, setting

$$
\mathbb{P}_{1}:=\bigcup_{k} \mathcal{P}_{1}^{k}
$$

we end the construction of the family of tiles having mass of order 1 .

\footnotetext{
${ }^{13}$ Notice that $\left\|\mathcal{N}_{1}^{0}\right\|_{\mathrm{BMO}_{D}(\mathbb{R})} \leq 2\left\|\mathcal{N}_{1}^{0}\right\|_{\mathrm{BMO}_{C}}$.
} 
Now suppose that we have constructed the sets $\left\{\mathbb{P}_{k}\right\}_{k<n}$. Here is how we define the set $\mathbb{P}_{n}$. First, select the family $\mathcal{P}_{n}^{0, \text { max }}$ of maximal tiles $P \in \mathbb{P}$ with $|E(P)| /\left|I_{P}\right| \geq 2^{-n}$. Then, collect the time intervals of these maximal tiles into the set $\mathcal{I}_{n}^{0}$ and define the counting function

$$
\mathcal{N}_{n}^{0}:=\sum_{I \in \mathcal{I}_{n}^{0}} \chi_{I}
$$

Next use John-Nirenberg inequality to get that

$$
A_{n}^{1}:=\left\{x \in[0,1]: \sum_{I \in \mathcal{I}_{n}^{0}} \chi_{I}(x)>c\left\|\mathcal{N}_{n}^{0}\right\|_{\mathrm{BMO}_{C}}\right\}
$$

has measure less than $1 / 2$ for an appropriate choice of $c$.

Proceeding as in the algorithm for the tiles of mass 1, we repeat the above procedure and construct

- the collection of sets of maximal tiles $\left\{\mathcal{P}_{n}^{k, \max }\right\}_{k}$;

- the collection of sets representing the time intervals $\left\{\mathcal{I}_{n}^{k}\right\}_{k}$;

- the collection of counting functions $\left\{\mathcal{N}_{n}^{k}\right\}_{k}$;

- the level sets $\left\{A_{n}^{k}\right\}_{k}$.

Notice the following key properties of our construction:

- $A_{n}^{k} \subset A_{n}^{l}$ and $\left|A_{n}^{k}\right| \leq 2^{-(k-l)}\left|A_{n}^{l}\right|$ for any $k \geq l$;

- $\sup _{k}\left\|\mathcal{N}_{n}^{k}\right\|_{\mathrm{BMO}_{C}} \leq 2^{n}$ and $\sup _{k}\left\|\mathcal{N}_{n}^{k}\right\|_{L^{\infty}\left(A_{n}^{k} \backslash A_{n}^{k+1}\right)} \lesssim 2^{n}$;

- if we set $\mathcal{N}_{n}:=\sum_{k} \mathcal{N}_{n}^{k}$, then $\left\|\mathcal{N}_{n}\right\|_{\mathrm{BMO}_{C}} \lesssim 2^{n}$.

Now for each $k \in \mathbb{N}$ set

$$
\mathcal{P}_{1}^{k}:=\left\{P=[\omega, I]: \begin{array}{c}
I \subseteq A_{n}^{k}, A_{\mathbb{P}, A_{n}^{k}}(P) \in\left[2^{-n}, 2^{-n+1}\right) \text { and } \\
\text { if } I \subseteq A_{n}^{k+1} \text { then } A_{\mathbb{P}, A_{n}^{k+1}}(P) \notin\left[2^{-n}, 2^{-n+1}\right]
\end{array}\right\} .
$$

and let

$$
\mathbb{P}_{n}:=\bigcup_{k \geq 0} \mathcal{P}_{n}^{k}
$$

Conclude that

$$
\mathbb{P}=\bigcup_{n \geq 0} \mathbb{P}_{n}
$$

This ends the mass partition of our set $\mathbb{P}$.

In [14], the author shows that each family $\mathbb{P}_{n}$ may be decomposed as a union of $C n\left(C \in \mathbb{N}\right.$ some absolute constant) BMO-forests of the $n^{\text {th }}$ generation. While this is not a problem if one aims for $L^{p}$ bounds $(1<p<\infty)$, when $p=1$ this becomes a serious issue. Still, by carefully inspecting the proof in [14], one can actually be more precise: $\mathbb{P}_{n}$ may be decomposed in at most $C$ sets $\bigcup_{l=1}^{n} \mathbb{P}_{n, l}$ with $\left\{\mathbb{P}_{n, l}\right\}_{l}$ a 
"special" sequence of BMO-forests such that: for each $l \in \mathbb{N}$, applying the decomposition in Definition 4.6, one can write $\mathbb{P}_{n, l}=\bigcup_{k} \mathbb{P}_{n, l}^{k}$, with $\mathbb{P}_{n, l}^{k}$ an $L^{\infty}$-forest having the $L^{\infty}$-norm of the counting function $\lesssim 2^{l}$. This fact resolves the possible difficulty and thus, from now on, we will always suppose that $\mathbb{P}_{n}$ stands for a BMO-forest of the $n^{\text {th }}$ generation.

\subsection{The Calderón-Zygmund decomposition: $\alpha$ discretization}

Unlike the mass decomposition, the Calderón-Zygmund decomposition presented below depends on the environment, namely on the function to which we apply the Carleson operator $T$.

Thus, once and for all, fix a nonzero function $f \in L^{1}$. Now choose $n \in \mathbb{N}$ and focus on the BMO-forest $\mathbb{P}_{n}$. Our aim is to write

$$
\mathbb{P}_{n}=\bigcup_{\alpha \in \mathbb{Z}} \mathbb{P}_{n}^{\alpha}
$$

with each $\mathbb{P}_{n}^{\alpha}$ being "nicely" structured and, in particular, satisfying (1.5).

We start by decomposing the torus into the corresponding level sets of the (dyadic) maximal function associated to $f$. First notice that without loss of generality we may assume that there exists $N \in \mathbb{Z}$ such that

$$
2^{N}<\|M f\|_{\infty} \leq 2^{N+1}
$$

On the other hand, since our function $f \in L^{1}(\mathbb{T})$ is nonzero,

$$
2^{M+1} \geq \frac{\int_{\mathbb{T}}|f|}{|\mathbb{T}|}>2^{M} \quad \text { for some } N \geq M \in \mathbb{Z} .
$$

Now let $\mathcal{J}_{\alpha}$ be the collection of maximal dyadic intervals $J$ such that

$$
\frac{\int_{J}|f|}{|J|}>2^{-\alpha}
$$

and let $\overline{\mathcal{J}}_{\alpha}:=\bigcup_{J \in \mathcal{J}_{\alpha}} J=\left\{M f>2^{-\alpha}\right\}$. We then have that

- for all $\alpha \in \mathbb{Z}$ such that $\alpha \leq-(N+1)$, we have $\overline{\mathcal{J}}_{\alpha}=\emptyset$;

- if $-N \leq \alpha_{1}<\alpha_{2} \leq-M$, then $\overline{\mathcal{J}}_{\alpha_{1}} \subsetneq \overline{\mathcal{J}}_{\alpha_{2}}$;

- for all $\alpha \in \mathbb{Z}$ such that $\alpha \geq-(M+1)$, we have $\overline{\mathcal{J}}_{\alpha}=\overline{\mathcal{J}}_{-M}=\mathbb{T}$.

For decomposing our family $\mathbb{P}_{n}$ we follow now an (increasing) inductive process. First set

$$
\mathbb{P}_{n}^{-N}:=\left\{P \in \mathbb{P}_{n}: \exists J \in \mathcal{J}_{-N} \text { such that } I_{P} \cap 51 J \neq \emptyset \quad \& \quad\left|I_{P}\right| \leq|J|\right\} .
$$

Suppose that we have constructed the set $\mathbb{P}_{n}^{\alpha-1}$. Then let

$$
\overline{\mathbb{P}}_{n}^{\alpha}:=\mathbb{P}_{n} \backslash \bigcup_{j \leq \alpha-1} \mathbb{P}_{n}^{j}
$$

and define

$$
\mathbb{P}_{n}^{\alpha}:=\left\{P \in \overline{\mathbb{P}}_{n}^{\alpha}: \exists J \in \mathcal{J}_{\alpha} \text { such that } I_{P} \cap 51 J \neq \emptyset \quad \& \quad\left|I_{P}\right| \leq|J|\right\} .
$$


By (5.7), the decomposition process will end in a finite number of steps since

- for all $\alpha \in \mathbb{Z}$ such that $\alpha \leq-(N+1)$,we can set $\mathbb{P}_{n}^{\alpha}=\emptyset$;

- for all $\alpha \in \mathbb{Z}$ such that $\alpha \geq-(M+1)$, we have $\mathbb{P}_{n}^{\alpha}=\emptyset$.

Thus, we obtain a partition

$$
\mathbb{P}_{n}=\bigcup_{\alpha \in \mathbb{Z}} \mathbb{P}_{n}^{\alpha}
$$

of the collection $\mathbb{P}_{n}$ such that

$$
\operatorname{supp} \mathbb{P}_{n}^{\alpha}:=\bigcup_{P \in \mathbb{P}_{n}^{\alpha}} I_{P} \subset 100\left\{M f>2^{-\alpha}\right\} .
$$

We end this section with several important observations.

Observation. 1) This partition of $P_{n}$ conserves the convexity property on which the boundedness of the trees depends heavily. More precisely we have that

$$
\text { if } P_{1}<P_{2}<P_{3} \text { such that } P_{1}, P_{3} \in \mathbb{P}_{n}^{\alpha} \text {, then } P_{2} \in \mathbb{P}_{n}^{\alpha} \text {. }
$$

2) Notice that for any $P \in \mathbb{P}_{n}^{\alpha} \neq \emptyset$ we have that

- either for all $J \in \mathcal{J}_{\alpha-1}, I_{P} \cap 51 J=\emptyset$,

- or if $J \in \mathcal{J}_{\alpha-1}$ is such that $I_{P} \cap 51 J \neq \emptyset$, then $\left|I_{P}\right|>|J|$.

3) For $P=\left[\omega_{P}, I_{P}\right] \in \mathbb{P}_{n}^{\alpha} \neq \emptyset$, let $c\left(I_{P}\right)$ be the center of the interval $I_{P}$ and define

$$
I_{P^{*}}=\left[c\left(I_{P}\right)-\frac{17}{2}\left|I_{P}\right|, c\left(I_{P}\right)-\frac{3}{2}\left|I_{P}\right|\right] \cup\left[c\left(I_{P}\right)+\frac{3}{2}\left|I_{P}\right|, c\left(I_{P}\right)+\frac{17}{2}\left|I_{P}\right|\right] .
$$

Then we have that

$$
\operatorname{supp} T_{P} \subseteq I_{P} \quad \text { and } \operatorname{supp} T_{P}^{*} \subseteq I_{P^{*}}
$$

Moreover, writing

$$
I_{P^{*}}=\bigcup_{r=1}^{14} I_{P *}^{r}
$$

with each $I_{P^{*}}^{r}$ a dyadic interval of length $\left|I_{P}\right|$, we have the property

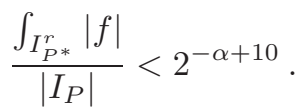

4) Let $\mathcal{P} \in \mathbb{P}_{n}^{\alpha}$ be a tree. Define $\mathcal{P}^{\text {min }}$ to be the collection of minimal tiles in $\mathcal{P}$. Furthermore, set

$$
\mathcal{J}_{\mathcal{P}^{*}}:=\left\{I_{P *}^{r}: P \in \mathcal{P}^{\text {min }} \text { and } r \in\{1, \ldots, 14\}\right\},
$$

and let $C Z\left(\mathcal{J}_{\mathcal{P}^{*}}\right)$ be the Calderón-Zygmund decomposition of the interval $[0,1]$ with respect to $\mathcal{J}_{\mathcal{P}^{*}}$.

Then, from (5.9) and (5.15) we deduce the following key property:

$$
\frac{\int_{I}|f|}{|I|}<2^{-\alpha+10} \quad \forall I \in C Z\left(\mathcal{J}_{\mathcal{P}^{*}}\right) .
$$




\section{The proof of the Theorem 1.1}

\subsection{Proof of a)}

With the notations and definitions previously introduced, our aim is to show that

$$
\operatorname{supp} T^{\mathbb{P}_{n}^{\alpha}} \subseteq 100\left\{M f>2^{-\alpha}\right\} \quad \text { and } \quad\left\|T^{\mathbb{P}_{n}^{\alpha}} f\right\|_{1} \lesssim 2^{-\alpha}\left|\left\{M f>2^{-\alpha}\right\}\right| .
$$

The first of the above conditions is an immediate consequence of the construction of the tile families $\left\{\mathbb{P}_{n}^{\alpha}\right\}$.

For the second condition we need to analyze the structure of each $\mathbb{P}_{n}^{\alpha}$. Let $\mathbb{P}_{n}^{\alpha}=$ $\bigcup_{j \in \mathbb{N}} \mathbb{P}_{n}^{\alpha, j}$ be the decomposition of $\mathbb{P}_{n}^{\alpha}$ into $L^{\infty}$-forests. Furthermore, for $j \in \mathbb{N}$, we decompose each $L^{\infty}$-forest $\mathbb{P}_{n}^{\alpha, j}=\bigcup_{k} \mathcal{P}_{k}^{\alpha, n, j}$ in maximal trees. Let $I_{P_{k}^{\alpha, n, j}}$ be the time interval of the top of the tree $\mathcal{P}_{k}^{\alpha, n, j}$ and define $I_{\mathbb{P}_{n}^{\alpha, j}}:=\bigcup_{k} I_{P_{k}^{\alpha, n, j}}$ and $I_{\mathbb{P}_{n}^{\alpha}}:=\bigcup_{j} I_{\mathbb{P}_{n}^{\alpha, j}}$ respectively.

Now, as a consequence of the construction in Section 5, for $\mathcal{N}_{j}^{\alpha, n}:=\sum_{k} \chi_{I_{P_{k}^{\alpha, n, j}}}$ and $\mathcal{N}^{\alpha, n}=\sum_{j} \mathcal{N}_{j}^{\alpha, n}$, we have

$$
\left\|\mathcal{N}_{j}^{\alpha, n}\right\|_{\infty} \lesssim 2^{n} \forall j \in \mathbb{N} \text { and }\left\|\mathcal{N}^{\alpha, n}\right\|_{1} \lesssim 2^{n}\left|I_{\mathbb{P}_{n}^{\alpha}}\right|
$$

By (6.2), it is thus enough to prove that for $\mathcal{P} \subset \mathbb{P}_{n}^{\alpha}$,

$$
\int\left|T^{\mathcal{P}} f\right| \lesssim 2^{-n} 2^{-\alpha}\left|I_{\mathcal{P}}\right|
$$

Indeed, from (6.3) and (6.2) we deduce

$$
\int\left|T_{n}^{\mathbb{P}_{n}^{\alpha}} f\right| \lesssim 2^{-n} 2^{-\alpha}\left\|\mathcal{N}^{\alpha, n}\right\|_{1} \lesssim 2^{-\alpha}\left|I_{\mathbb{P}_{n}^{\alpha}}\right| \lesssim 2^{-\alpha}\left|\left\{M f>2^{-\alpha}\right\}\right| .
$$

Now to show (6.3) we proceed as follows.

Without loss of generality we may assume that all the tiles $P \in \mathcal{P}$ are at a constant frequency $\omega$ (the frequency of the tree).

Define

$$
\mathcal{L}_{\mathcal{P}}(f):=\sum_{J \in C Z\left(\mathcal{J}_{\mathcal{P}} *\right)} \frac{\int_{J} f(s) e^{i \omega s} d s}{|J|} \chi_{J}
$$

Observe that as a consequence of (5.16) we have

$$
\left|\mathcal{L}_{\mathcal{P}}(f)\right| \lesssim 2^{-\alpha} \chi_{I_{\mathcal{P}}} .
$$

Now letting $\mathcal{P}_{0}$ be the shift of $\mathcal{P}$ to the origin, we have

$$
\int\left|T^{\mathcal{P}} f\right| \leq \int\left|T^{\mathcal{P}_{0}}\left(e^{i \omega \cdot} f(\cdot)-\mathcal{L}_{\mathcal{P}}(f)(\cdot)\right)\right|+\int\left|T^{\mathcal{P}_{0}}\left(\mathcal{L}_{\mathcal{P}}(f)(\cdot)\right)\right| .
$$

For the first term we use the mean zero condition

$$
\int_{I_{P^{*}}}\left\{f(\cdot) e^{i \omega \cdot}-\mathcal{L}_{\mathcal{P}}(f)(\cdot)\right\}=0 \quad \forall P \in \mathcal{P},
$$


and deduce that

$$
\int\left|T^{\mathcal{P}_{0}}\left(e^{i \omega} \cdot f(\cdot)-\mathcal{L}_{\mathcal{P}}(f)(\cdot)\right)\right| \lesssim 2^{-n}\|f\|_{L^{1}\left(I_{\mathcal{P}}\right)}
$$

For the second term, we use the relation (6.6) and the $L^{2}$-boundedness of the Hilbert transform:

$$
\int\left|T^{\mathcal{P}_{0}}\left(\mathcal{L}_{\mathcal{P}}(f)\right)\right| \lesssim|E(\mathcal{P})|^{1 / 2} 2^{-n / 2}\left\|\mathcal{L}_{\mathcal{P}}(f)\right\|_{L^{2}\left(I_{\mathcal{P}}\right)} \lesssim 2^{-n} 2^{-\alpha}\left|I_{\mathcal{P}}\right|
$$

where we set $E(\mathcal{P}):=\bigcup_{P \in \mathcal{P}} E(P)$. Here we have used the key Carleson measure estimate

$$
|E(\mathcal{P})| \lesssim 2^{-n}\left|I_{\mathcal{P}}\right|
$$

Indeed, for proving (6.9), we follow the reasoning from [13] (page 481) and for

$$
\mathcal{J}_{\mathcal{P}}:=\left\{I_{P}: P \in \mathcal{P}^{\min }\right\},
$$

we set

$$
\begin{aligned}
\check{\mathcal{J}}(\mathcal{P}):=\left\{I \subset I_{\mathcal{P}}:\right. \text { exactly one of the left or right halves } \\
\text { of } \left.I \text { contains an element of } \mathcal{J}_{\mathcal{P}}\right\} \cup \mathcal{J}_{\mathcal{P}} .
\end{aligned}
$$

and $\breve{\mathcal{P}}=\{P=[\omega, I] \in \mathcal{P}: I \in \check{\mathcal{J}}(\mathcal{P})\}$. Then we have

$$
|E(\mathcal{P})| \leq \sum_{P \in \breve{\mathcal{P}}}|E(P)| \lesssim 2^{-n} \sum_{I \in \breve{\mathcal{J}}(\mathcal{P})}|I| \lesssim 2^{-n}\left|I_{\mathcal{P}}\right|
$$

This ends our proof. Notice that (6.1) implies

$$
\left\|T^{\mathbb{P}_{n}}\right\|_{1} \lesssim \sum_{\alpha}\left\|T^{\mathbb{P}_{n}^{\alpha}} f\right\|_{1} \lesssim\|M f\|_{1} \lesssim\|f\|_{L \log L}
$$

\subsection{Proof of $b$ )}

In this section we will show a slightly stronger statement than the one claimed in Theorem 1.1. More precisely, we prove that the operators $\left\{T^{\mathbb{P}_{n}}\right\}_{n}$ are uniformly weak $(1,1)$ bounded.

We claim that for all $G \subseteq[0,1]$ there exists $G^{\prime} \subseteq G,\left|G^{\prime}\right|>\frac{1}{2}|G|$, such that ${ }^{14}$

$$
\int_{G^{\prime}}\left|T^{\mathbb{P}_{n}} f\right| \lesssim\|f\|_{1} \quad \forall n \in \mathbb{N}
$$

\footnotetext{
${ }^{14} \mathrm{~A}$ similar statement for a rougher mass-discretized family $\mathbb{P}_{n}$ is proved in [8]. For this, the authors use the mass-size decomposition technique presented in [12]. Then they interpolate the resulting $L^{1}$-estimate with a "modified" $L^{2}$-estimate to get good control near $L^{1}$. This interpolation reasoning is also used in our approach.
} 
Fix $G \subset \mathbb{T}$ and define $G^{\prime}$ by

$$
G^{\prime}=\left\{x \in G: M f(x) \leq C \frac{\|f\|_{1}}{|G|}\right\}
$$

where here $C>0$ is some large appropriately chosen constant. This guarantees that $G^{\prime} \subset G$, with $\left|G^{\prime}\right| \gtrsim|G|$.

Set now $\lambda=C\|f\|_{1} /|G|$. It will be enough to prove the following: ${ }^{15}$

Lemma 6.1. Let $k \in \mathbb{N}$ and suppose $2^{-\alpha} \approx \lambda 2^{-k}$. Then the following relation holds:

$$
\int_{G^{\prime}}\left|T^{\mathbb{P}_{n}^{\alpha}} f\right| \lesssim 2^{-k / 2}\|f\|_{1} \quad \forall n \in \mathbb{N}
$$

This result relies on the tree estimate provided below.

Lemma 6.2. Let $\mathcal{P} \subset \mathbb{P}_{n}^{\alpha}$ be a tree with top $I_{\mathcal{P}}$. Then we have

$$
\int_{G^{\prime}}\left|T^{\mathcal{P}} f\right| \lesssim 2^{-\alpha}\left|G^{\prime} \cap E(\mathcal{P})\right|^{1 / 2} 2^{-n / 2}\left|I_{\mathcal{P}}\right|^{1 / 2} .
$$

Proof. Let $C Z\left(\mathcal{J}_{\mathcal{P}^{*}}\right)$ be the Calderón-Zygmund decomposition described in the observation 4) of Section 5.2. As before, we can assume ${ }^{16}$ without loss of generality that all the tiles $P \in \mathcal{P}$ are at a constant frequency $\omega=0$.

Now following the description from a) we have

$$
\int_{G^{\prime}}\left|T^{\mathcal{P}} f\right| \leq \int_{G^{\prime}}\left|T^{\mathcal{P}}\left(f-\mathcal{L}_{\mathcal{P}}(f)\right)\right|+\int_{G^{\prime}}\left|T^{\mathcal{P}}\left(\mathcal{L}_{\mathcal{P}}(f)\right)\right|
$$

The second term is trivially bounded by the Cauchy-Schwarz inequality and the $L^{2}$ boundedness of the Hilbert transform:

$$
\begin{aligned}
\int_{G^{\prime}}\left|T^{\mathcal{P}}\left(\mathcal{L}_{\mathcal{P}}(f)\right)\right| & \lesssim\left|G^{\prime} \cap E(\mathcal{P})\right|^{1 / 2} 2^{-n / 2}\left\|\mathcal{L}_{\mathcal{P}}(f)\right\|_{L^{2}\left(I_{\mathcal{P}}\right)} \\
& \lesssim 2^{-\alpha}\left|G^{\prime} \cap E(\mathcal{P})\right|^{1 / 2} 2^{-n / 2}\left|I_{\mathcal{P}}\right|^{1 / 2} .
\end{aligned}
$$

For the first term we need to be more careful; we will show that for any $g \in$ $L^{\infty}(\mathbb{T})$ with supp $g \subseteq G^{\prime}$ we have

$$
\left|\int\left(T^{\mathcal{P}^{*}} g\right)\left(f-\mathcal{L}_{\mathcal{P}}(f)\right)\right| \lesssim 2^{-\alpha}\left|G^{\prime} \cap E(\mathcal{P})\right|^{1 / 2} 2^{-n / 2}\left|I_{\mathcal{P}}\right|^{1 / 2}\|g\|_{\infty} .
$$

At this point we make essential use of the mean zero property

$$
\int_{J}\left(f-\mathcal{L}_{\mathcal{P}}(f)\right)=0 \quad \forall J \in C Z\left(\mathcal{J}_{\mathcal{P}^{*}}\right) .
$$

\footnotetext{
${ }^{15}$ For two positive quantities $A$ and $B$ we write $A \approx B$ if $2^{-10} B<A<2^{10} B$.

${ }^{16}$ To put our problem in the context of this assumption we take advantage of the translation invariance of our statement and use a standard estimate (see [9]) that gives an error of order $2^{-\alpha}\left|G^{\prime} \cap E(\mathcal{P})\right|$.
} 
Thus, for proving (6.14), it is enough to show that, for $g=g \chi_{G^{\prime}} \in L^{\infty}$,

$$
\int\left|\left(T^{\mathcal{P}^{*}} g-\mathcal{L}_{\mathcal{P}}\left(T^{\mathcal{P}^{*}} g\right)\right)\left(f-\mathcal{L}_{\mathcal{P}}(f)\right)\right| \lesssim 2^{-\alpha}\left|G^{\prime} \cap E(\mathcal{P})\right|^{1 / 2} 2^{-n / 2}\left|I_{\mathcal{P}}\right|^{1 / 2}\|g\|_{\infty}
$$

For fixed $J \in C Z\left(\mathcal{J}_{\mathcal{P}^{*}}\right)$ and $x \in J$ we have

$$
\begin{aligned}
\mid T^{\mathcal{P}^{*}} g(x)- & \frac{1}{|J|} \int_{J} T^{\mathcal{P}^{*}} g(s) d s \mid \\
& =\left|\frac{1}{|J|} \int_{J}\left\{\sum_{\substack{P \in \mathcal{P} \\
\left|I_{P}\right| \geq|J|}} \int_{\mathbb{T}}\left[\varphi_{k}(x-y)-\varphi_{k}(s-y)\right] g(y) \chi_{E(P)}(y) d y\right\} d s\right| \\
& \lesssim \frac{1}{|J|} \int_{J}\left\{\sum_{I_{P * \supseteq J}}\left|I_{P}\right|^{-1}|J| \frac{\int_{E(P) \cap G^{\prime}}|g|}{\left|I_{P}\right|}\right\} d s .
\end{aligned}
$$

Thus, we have proved

$$
\left|T^{\mathcal{P}^{*}} g(x)-\frac{1}{|J|} \int_{J} T^{\mathcal{P}^{*}} g(s) d s\right| \chi_{J} \lesssim\|g\|_{\infty} \frac{\chi_{J}}{|J|} \sum_{I_{P^{*} \supseteq J}} \frac{|J|^{2}}{\left|I_{P}\right|^{2}}\left|G^{\prime} \cap E(P)\right| .
$$

This last relation gives us

$$
\begin{aligned}
\int \mid\left(T^{\mathcal{P}^{*}} g-\right. & \left.\mathcal{L}_{\mathcal{P}}\left(T^{\mathcal{P}^{*}} g\right)\right)\left(f-\mathcal{L}_{\mathcal{P}}(f)\right) \mid \\
& \lesssim 2^{-\alpha} 2^{-n / 2}\|g\|_{\infty} \sum_{J \in C Z\left(\mathcal{J}_{\mathcal{P}}\right)} \sum_{I_{P^{*}} \supseteq J}|J|^{1 / 2}\left(|J| /\left|I_{P}\right|\right)^{3 / 2}\left|G^{\prime} \cap E(P)\right|^{1 / 2} \\
& \lesssim 2^{-\alpha}\left|G^{\prime} \cap E(\mathcal{P})\right|^{1 / 2} 2^{-n / 2}\left|I_{\mathcal{P}}\right|^{1 / 2}\|g\|_{\infty},
\end{aligned}
$$

proving (6.12). Here we have relied on the following key relation:

$$
\sum_{P \in \mathcal{P}} \sum_{\substack{J \in C Z\left(\mathcal{J}_{\mathcal{P}}^{*}\right) \\ J \subseteq I_{P^{*}}}}\left(\frac{|J|}{\left|I_{P}\right|}\right)^{3 / 2}|J|^{1 / 2}\left|G^{\prime} \cap E(P)\right|^{1 / 2} \lesssim\left|G^{\prime} \cap E(\mathcal{P})\right|^{1 / 2}\left|I_{\mathcal{P}}\right|^{1 / 2}
$$

To prove (6.17), take $l \in \mathbb{N}$ and set

$$
C Z_{l}^{*}\left(I_{P}\right):=\left\{J \in C Z\left(\mathcal{J}_{\mathcal{P}^{*}}\right)\left|J \subseteq I_{P^{*}}, \quad\right| J\left|\approx 2^{-l}\right| I_{P} \mid\right\} .
$$

Now (6.17) will be a consequence of

$$
S_{l}:=\sum_{P \in \mathcal{P}} \sum_{J \in C Z_{l}^{*}\left(I_{P}\right)}|J|^{1 / 2}\left|G^{\prime} \cap E(P)\right|^{1 / 2} \lesssim \epsilon 2^{l(1 / 2+\epsilon)}\left|G^{\prime} \cap E(\mathcal{P})\right|^{1 / 2}\left|I_{\mathcal{P}}\right|^{1 / 2}
$$

where here $\epsilon \in(0,1)$ is some absolute constant.

To prove (6.18), we start by refining the set $\mathcal{P}$ as follows: for $u \in\{0 \ldots, l+100\}$, let

$$
\mathcal{P}^{l, u}:=\left\{P \in \mathcal{P}: 2^{u-1}<\# C Z_{l}^{*}\left(I_{P}\right) \leq 2^{u}\right\} .
$$

Fix $u$ and define

$$
S_{l, u}:=\sum_{P \in \mathcal{P} l, u} \sum_{J \in C Z_{l}^{*}\left(I_{P}\right)}|J|^{1 / 2}\left|G^{\prime} \cap E(P)\right|^{1 / 2} .
$$


Next, we decompose the set $\mathcal{P}^{l, u}$ inductively as follows: let $\mathcal{P}_{1}^{l, u}$ be the collection of maximal (with respect to " $\leq$ ") tiles in $\mathcal{P}^{l, u}$; repeat this procedure for the collection $\mathcal{P}^{l, u} \backslash \mathcal{P}_{1}^{l, u}$ and thus construct the set of maximal tiles $\mathcal{P}_{2}^{l, u}$. Continue this process until $\mathcal{P}^{l, u}$ is exhausted. We finish with a partition

$$
\mathcal{P}^{l, u}=\bigcup_{r=1}^{m} \mathcal{P}_{r}^{l, u}
$$

of collections of (successively) maximal tiles.

Applying now Cauchy-Schwarz we deduce that

$$
S_{l, u} \leq\left|G^{\prime} \cap E(\mathcal{P})\right|^{1 / 2} \sum_{r=1}^{m}\left(\sum_{P \in \mathcal{P}_{r}^{l, u}}\left(\sum_{J \in C Z_{l}^{*}\left(I_{P}\right)}|J|^{1 / 2}\right)^{2}\right)^{1 / 2}
$$

Finally, we observe that

$$
\sum_{r=1}^{m}\left(\sum_{P \in \mathcal{P}_{r}^{l, u}}\left(\sum_{J \in C Z_{l}^{*}\left(I_{P}\right)}|J|^{1 / 2}\right)^{2}\right)^{1 / 2} \lesssim 2^{l / 2}\left|I_{\mathcal{P}}\right|^{1 / 2}
$$

This last fact is a consequence of the construction of the sets $\left\{\mathcal{P}_{r}^{l, u}\right\}$, the definition of $C Z_{l}^{*}\left(I_{P}\right)$ and the fact that for any $J \in C Z\left(\mathcal{J}_{\mathcal{P}^{*}}\right)$ one has

$$
\#\left\{P \in \mathcal{P}: J \in C Z_{l}^{*}\left(I_{P}\right)\right\} \lesssim 1 .
$$

Indeed, by the above mentioned facts, one has that the main contribution to the left hand side of (6.20) comes from the first $O\left(2^{l-u}\right)$ terms of the sum. We leave further details to the interested reader.

We pass now to the proof of Lemma 6.1. With the notations from point a) and by (6.12), for each $L^{\infty}$-forest $\mathbb{P}_{n}^{\alpha, j}$ we decompose $\mathbb{P}_{n}^{\alpha, j}=\bigcup_{k} \mathcal{P}_{k}^{\alpha, n, j}$ into maximal trees and deduce

$$
\begin{aligned}
\int_{G^{\prime}}\left|T^{\mathbb{P}_{n}^{\alpha, j}} f\right| & \lesssim \sum_{k \in \mathbb{N}} \int_{G^{\prime}}\left|T^{\mathcal{P}_{k}^{\alpha, n, j}} f\right| \lesssim 2^{-\alpha} \sum_{k \in \mathbb{N}}\left|G^{\prime} \cap E\left(\mathcal{P}_{k}^{\alpha, n, j}\right)\right|^{1 / 2} 2^{-n / 2}\left|I_{\mathcal{P}_{k}^{\alpha, n, j}}\right|^{1 / 2} \\
& \leq 2^{-\alpha} 2^{-n / 2}\left\{\sum_{k \in \mathbb{N}}\left|G^{\prime} \cap E\left(\mathcal{P}_{k}^{\alpha, n, j}\right)\right|\right\}^{1 / 2}\left\{\sum_{k \in \mathbb{N}}\left|I_{\mathcal{P}_{k}^{\alpha, n, j}}\right|\right\}^{1 / 2} \\
& \lesssim 2^{-\alpha}\left|G^{\prime} \cap E\left(\mathbb{P}_{n}^{\alpha, j}\right)\right|^{1 / 2}\left|I_{\mathbb{P}_{n}^{\alpha, j}}\right|^{1 / 2}
\end{aligned}
$$

From the Carleson measure condition imposed in Definition 4.6 ii), we have

$$
\int_{G^{\prime}}\left|T^{\mathbb{P}_{n}^{\alpha}} f\right| \lesssim \sum_{j} \int_{G^{\prime}}\left|T^{\mathbb{P}_{n}^{\alpha, j}} f\right| \lesssim 2^{-\alpha}\left|G^{\prime}\right|^{1 / 2} \sum_{j}\left|I_{\mathbb{P}_{n}^{\alpha, j}}\right|^{1 / 2} \lesssim 2^{-\alpha}\left|G^{\prime}\right|^{1 / 2}\left|I_{\mathbb{P}_{n}^{\alpha}}\right|^{1 / 2}
$$

Finally, using that

$$
\left|I_{\mathbb{P}_{n}^{\alpha}}\right| \lesssim\left|\overline{\mathcal{J}}_{\alpha}\right| \approx 2^{\alpha} \int_{\overline{\mathcal{J}}_{\alpha}}|f|
$$

and setting $2^{-\alpha} \approx \lambda 2^{-k}$ we conclude that (6.11) holds. 


\subsection{Proof of $\mathrm{c}$ )}

The central estimate for proving (1.9) is given by

$$
\left\|T^{\mathbb{P}_{n}} f\right\|_{2} \lesssim n^{2} 2^{-n / 2}\|f\|_{2}
$$

We limit ourselves to providing the main ideas. For the details of the proof the reader should consult [14]. One first decomposes $\mathbb{P}_{n}=\bigcup \mathbb{P}_{n}^{j}$ with each $\mathbb{P}_{n}^{j}$ an $L^{\infty}$-forest, and then proves that

- $\left\|T^{\mathbb{P}_{n}^{j}} f\right\|_{2} \lesssim n 2^{-n / 2}\|f\|_{2}$.

- for each $k \in\{0, \ldots, n-1\}$ the sequence $\left\{T^{\mathbb{P}_{n}^{k+j n}}\right\}_{j \in \mathbb{N}}$ comprises almost orthogonal operators and thus one can apply the Cotlar-Stein lemma.

This ensures that (6.22) holds. Now, for $1<p \leq 2,(1.9)$ is just a consequence of (1.8), (6.22) and classical interpolation theory. For $p>2$ we can proceed as in the $L^{2}$ case: one first proves the desired estimate for an $L^{\infty}$ forest and then using the structure of the BMO-forest one is able to extend the initial result to the entire family $\mathbb{P}_{n}$ (for more details see $[14]$ ).

\subsection{Proof of $\mathrm{d}$ )}

We will show ${ }^{17}$ that, for $1<p<\infty$,

$$
\|T f\|_{1, \infty} \lesssim p\|f\|_{1} \log \frac{2\|f\|_{p}}{\|f\|_{1}} .
$$

As before, we start by reformulating (6.23) in the equivalent form: for all $G \subset \mathbb{T}$ there exists $G^{\prime} \subset G$ with $\left|G^{\prime}\right| \gtrsim|G|$ such that

$$
\int_{G^{\prime}}|T f| \lesssim p\|f\|_{1} \log \frac{2\|f\|_{p}}{\|f\|_{1}}
$$

Repeat the construction as in a). Then, with the notations as in c), we have

$$
\int_{G^{\prime}}\left|T^{\mathbb{P}_{n}} f\right| \lesssim_{p} 2^{-\delta n\left(1-1 / p^{*}\right)}\|f\|_{p}
$$

Combining (1.8) and (6.25) we conclude that

$\int_{G^{\prime}}|T f| \leq \sum_{n \in \mathbb{N}} \int_{G^{\prime}}\left|T^{\mathbb{P}_{n}} f\right| \lesssim p \sum_{n \in \mathbb{N}} \min \left\{\|f\|_{1}, 2^{-\delta n\left(1-1 / p^{*}\right)}\|f\|_{p}\right\} \lesssim_{p}\|f\|_{1} \log \frac{2\|f\|_{p}}{\|f\|_{1}}$ proving the desired result.

\footnotetext{
${ }^{17}$ In [4], one can find (without proof) the following statement: for $1 \leq p \leq 2$ we have that $\|T f\|_{p, \infty} \lesssim\|f\|_{p} \log \frac{2\|f\|_{2}}{\|f\|_{p}}$. The unpublished proof ([3]) of this result, communicated to me by Arias de Reyna, relies on Carleson's original approach to the pointwise convergence of the Fourier series. Finally, note that the case $p=1$ in his result is equivalent with the case $p=2$ in (6.23).
} 


\section{Remarks}

1) As mentioned in the introduction, the central part of our paper is the tile decomposition described in Section 5.

The mass discretization of $T f$ is independent ${ }^{18}$ of $f$ and results in the geometric organization of $\mathbb{P}=\bigcup_{n} \mathbb{P}_{n}$ which gives us both the $n$-decay ${ }^{19}$ for each $\left\{\left\|T^{\mathbb{P}_{n}} f\right\|_{p}\right\}_{n}$ with $1<p<\infty\left(f \in L^{p}\right)$ and the ability to $\operatorname{sum}^{20}$ within each "scale" $n$ the lengths of the time support of the maximal trees in $\mathbb{P}_{n}$.

The Calderón-Zygmund discretization $\mathbb{P}_{n}=\bigcup_{\alpha} \mathbb{P}_{n}^{\alpha}$ realizes the decomposition of the function $T^{\mathbb{P}_{n}} f$ depending on $f$ and is designed to get good control near $L^{1}$. This discretization accounts for the multi-frequency nature of our problem. A similar issue appeared in [15], where the authors describe a "Calderón-Zygmund decomposition for multiple frequencies" for a given function $f$. Our approach though is quite different: instead of decomposing the input object (the function $f$ ) at multiple frequencies imposing a mean zero condition on the initial function for each frequency, we rely on the properties ${ }^{21}$ of the initial discretization $\mathbb{P}=\bigcup_{n} \mathbb{P}_{n}$ and first decompose the tile family $\mathbb{P}_{n}$ into subfamilies $\left\{\mathbb{P}_{n}^{\alpha}\right\}_{\alpha}$ followed by a further decomposition of the corresponding output object $T^{\mathbb{P}_{n}^{\alpha}} f$ into multiple pieces, with each piece having the mean zero condition strictly relative to the frequency at which it lives.

In the setting of the present paper our procedure is more effective and unlike the one in [15] gives an explicit construction. On the other hand, the decomposition in [15] is more general and hence can be used in other problems which are not necessarily related to the Carleson operator.

2) The treatment of the "forest" operators $T^{\mathbb{P}_{n}}$ in Theorem 1.1 offers a substitute for the classical theory of Calderón-Zygmund operators. Indeed, following the tile decomposition and the proofs of a) and b) one notices that the weak $(1,1)$ bound is obtained by using a Vitali type covering argument, in which we can sum the lengths of the intervals of the trees in the structure of $T^{\mathbb{P}_{n}} f$ depending on the size of the maximal function associated to $f$. Also, as in the classical theory, once we apply our tile decomposition, the entire difficulty resides in providing $L^{2}$ bounds on $T^{\mathbb{P}_{n}}$. For this last task, one needs to use orthogonality methods. To complete the parallelism, it would be of interest if our methods could provide a satisfactory theory for the adjoint operator $T^{\mathbb{P}_{n}}{ }^{*}$ near $L^{1}$. We think this topic deserves further investigation.

3) Finally, in view of our approach, the following question appears to be natural:

Open question. Fix $f \in L^{1}(\mathbb{T})$. With the previous notations and definitions, set

$$
\mathbb{P}^{\alpha}:=\bigcup_{n} \mathbb{P}_{n}^{\alpha}, \quad \text { with } \alpha \in \mathbb{N} .
$$

\footnotetext{
${ }^{18}$ The mass parameter depends on the function $N$ which may be taken as just an arbitrary measurable function as long as the final estimates on the operator $T$ do not depend on it.

${ }^{19}$ This helps us in both summing the operators $\left\{T^{\mathbb{P}_{n}}\right\}_{n}$ (and thus obtaining $L^{p}$ bounds for $1<p<\infty)$ and in interpolating with norm estimates near $L^{1}$.

${ }^{20}$ Here is the point where our technique overcomes the difficulty of treating exceptional sets.

${ }^{21}$ Here the algorithm described for the mass decomposition plays a fundamental role.
} 
Is it then true that there exists an absolute constant $C>0$ such that, for any $\alpha \in \mathbb{N}$,

$$
\left\|T^{\mathbb{P}^{\alpha}} f\right\|_{1} \leq C\|f\|_{1} ?
$$

A positive answer to this question would imply that

$$
\|T f\|_{L^{1}} \lesssim\|f\|_{L \log L}
$$

which is the best one can hope for if we require strong $L^{1}$ bounds for the Carleson operator $T$.

Of course it would be still very interesting if (7.1) holds with the $L^{1}$ norm replaced by the $L^{1, \infty}$ norm on the left-hand side. With the current technology we can only prove that

$$
\left\|T^{\mathbb{P}^{\alpha}} f\right\|_{1} \leq C\|f\|_{L \log L}
$$

\section{Appendix. Spaces near $L^{1}$}

In this section we briefly introduce the definitions of the relevant rearrangement invariant Banach spaces which appeared in the previous literature when studying the problem of the pointwise convergence of Fourier series near $L^{1}$.

Definition 8.1. Let $\varphi:[0, \infty) \mapsto[0, \infty)$ be an absolutely continuous function with the following properties:

- there is $C>0$ such that $\varphi\left(t^{2}\right) \leq C \varphi(t)$ for all $t \geq 0$;

- $\varphi^{\prime}(t) \geq 0$ almost everywhere;

- $\varphi(0)=0$;

- $\lim _{t \rightarrow \infty} \varphi(t)=\infty$.

Then $L \varphi(L)$ is the space of all (measurable) functions $f$ for which

$$
\|f\|_{\mathcal{L} \varphi(\mathcal{L})}:=\int_{\mathbb{T}}|f||\varphi(f)|<\infty
$$

A classical result in Banach space theory (see e.g. [5]) asserts:

Proposition. The space $L \varphi(L)$ endowed with the norm $^{22}$

$$
\|f\|_{L \varphi(L)}:=\int_{\mathbb{T}} f^{*}(t) \varphi(1 / t) d t<\infty,
$$

becomes a rearrangement-invariant Banach space.

In the topic treated in this paper three special choices for the function $\varphi$ are of interest:

- $\varphi(t)=\log (1+t)$ defines the Zygmund space $L \log L$;

\footnotetext{
${ }^{22}$ Here $f^{*}$ stands for the decreasing rearrangement of $f$.
} 
- $\varphi(t)=\log (1+t) \log \log (10+t)$ defines the space $L \log L \log \log L$ considered by Sjölin in [16].

- $\varphi(t)=\log (1+t) \log \log \log (10+t)$ defines the space $L \log L \log \log \log L$ considered by Antonov in [1].

To complete the picture, we need to consider two more spaces.

The first space was considered by F. Soria in [17]. Let $B_{\varphi}$ be the set of the measurable functions for which

$$
\|f\|_{\varphi}:=\int_{0}^{\infty} \varphi\left(\lambda_{f}(t)\right) d t<\infty,
$$

where $\lambda_{f}$ is the distribution function of $f$ given by $\lambda_{f}(t)=|\{x \in \mathbb{T}|| f(x) \mid>t\}|$. Take now the subspace $B_{\varphi}^{*} \subset B_{\varphi}$ defined by

$$
B_{\varphi}^{*}:=\left\{f:\|f\|_{\varphi}^{*}<\infty\right\}
$$

where

$$
\|f\|_{\varphi}^{*}=\int_{0}^{\infty} \varphi\left(\lambda_{f}(t)\right)\left(1+\log \left(\frac{\|f\|_{\varphi}}{\varphi\left(\lambda_{f}(t)\right)}\right)\right) d t .
$$

The pointwise convergence theory developed by Soria applied to the space $B_{\varphi_{1}}^{*}$ with $\varphi_{1}(s)=s\left(1+\log ^{+} 1 / s\right)$.

The second space was introduced by Arias de Reyna in [2]. Let $Q A$ be the quasi-Banach space defined as follows:

$$
\begin{aligned}
Q A & :=\left\{f: \mathbb{T} \mapsto C \mid f \text { measurable, }\|f\|_{Q A}<\infty\right\} \text { where } \\
\|f\|_{Q A} & :=\inf \left\{\sum_{j=1}^{\infty}(1+\log j)\left\|f_{j}\right\|_{1} \log \frac{2\left\|f_{j}\right\|_{\infty}}{\left\|f_{j}\right\|_{1}}\left|f=\sum_{j=1}^{\infty} f_{j}, \sum_{j=1}^{\infty}\right| f_{j} \mid<\infty \text { a.e. }\right\} .
\end{aligned}
$$

With the exception of Zygmund's $L \log L$ space, it is known ([16], [1], [17] and [2]) that all the other spaces considered here, namely $L \log L \log \log L, L \log L$ $\log \log \log L, B_{\varphi_{1}}^{*}$ and $Q A$ are rearrangement invariant spaces of functions with almost everywhere convergent Fourier series.

\section{References}

[1] Antonov, N. Yu.: Convergence of Fourier series. East J. Approx. 2 (1996), no. 2, 187-196.

[2] Arias de Reyna, J.: Pointwise convergence of Fourier series. J. London Math. Soc. (2) 65 (2002), no. 1, 139-153.

[3] Arias de Reyna, J.: About Carleson's Theorem. Unpublished.

[4] Arias de Reyna, J.: Pointwise convergence of Fourier series. Lecture Notes in Mathematics 1785, Springer-Verlag, Berlin, 2002.

[5] Bennett, C. And Sharpley, R.: Interpolation of operators. Pure and Applied Mathematics 129, Academic Press, Boston, MA, 1988. 
[6] Carleson L.: On convergence and growth of partial sumas of Fourier series. Acta Math. 116 (1966), 135-157.

[7] Carro, M., Mastylo, M. and Rodríguez-Piazza, L.: Almost everywhere convergent Fourier series. J. Fourier Anal. Appl. 18 (2012), no. 2, 266-286.

[8] Do, Y. And Lacey, M.: On the convergence of lacunary Walsh-Fourier series. Bull. London Math. Soc. 44 (2012), no. 2, 241-254.

[9] Fefferman, C.: Pointwise convergence of Fourier series. Ann. of Math. (2) 98 (1973), 551-571.

[10] Hunt, R. A.: On the convergence of Fourier series. In Orthogonal Expansions and their Continuous Analogues (Proc. Conf., Edwardsville, Ill., 1967), 235-255. Southern Illinois Univ. Press, Carbondale, Ill., 1968.

[11] Kalton, N. J.: Convexity, type and the three space problem. Studia Math. 69 (1980/81), no. 3, 247-287.

[12] Lacey, M. AND Thiele, C.: A proof of boundedness of the Carleson operator. Math. Res. Lett. 7 (2000), no. 4, 361-370.

[13] LiE, V.: The (weak- $L^{2}$ ) boundedness of the quadratic Carleson operator. Geom. Funct. Anal. 19 (2009), no. 2, 457-497.

[14] Lie, V.: The polynomial Carleson operator. Preprint, arXiv: 1105.4504v1, 2011.

[15] Nazarov, F., Oberlin, R. And Thiele, C.: A Calderón-Zygmund decomposition for multiple frequencies and an application to an extension of a lemma of Bourgain. Math. Res. Lett. 17 (2010), no. 3, 529-545.

[16] Sjölin, P.: An inequality of Paley and convergence a.e. of Walsh-Fourier series. Ark. Mat. 7 (1969), 551-570.

[17] Soria, F.: Note on differentiation of integrals and the halo conjecture. Studia Math. 81 (1985), no. 1, 29-36.

[18] Soria, F.: On an extrapolation theorem of Carleson-Sjölin with applications to a.e. convergence of Fourier series. Studia Math. 94 (1989), no. 3, 235-244.

[19] Stein, E. M.: On limits of seqences of operators. Ann. of Math. (2) 74 (1961), $140-170$.

Received December 18, 2011.

Victor LiE: Department of Mathematics, Princeton University, Fine Hall, Washington Road, Princeton, NJ 08544-1000, USA; and Institute of Mathematics of the Romanian Academy, Bucharest, RO 70700, P.O. Box 1-764, Romania.

E-mail: vlie@math.princeton.edu 